\title{
Effects of Far Infrared Light on High-Fat Diet-Treated Mice
}

\author{
Koji Fukui*1,2, Yugo Kato ${ }^{3}$ and Masahiro Kohno ${ }^{2,4}$ \\ ${ }^{1}$ Department of Bioscience and Engineering, Molecular Cell Biology Laboratory, College of Systems Engineering and Sciences, \\ Shibaura Institute of Technology (SIT), Japan \\ ${ }^{2}$ The Brain Science \& Life Technology Research Center, SIT Research Laboratories, SIT, Japan
}

${ }^{3}$ Department of Functional Control Systems, Molecular Cell Biology Laboratory, Graduate School of Engineering and Science, SIT, Japan

${ }^{4}$ Department of Life Science and Technology, Tokyo Institute of Technology, Japan

*Corresponding author: Koji Fukui, Molecular Cell Biology Laboratory, Department of Bioscience and Engineering, College of Systems Engineering and Sciences, Shibaura Institute of Technology, Fukasaku 307, Minuma-ku, Saitama, 3378570, Japan

\section{ARTICLE INFO}

Received: 彗 January 13, 2021

Published: 业 February 12, 2021

Citation: Koji Fukui, Yugo Kato, Masahiro Kohno. Effects of Far Infrared Light on High-Fat Diet-Treated Mice. Biomed J Sci \& Tech Res 33(5)-2021. BJSTR. MS.ID.005468.

Keywords: Far infrared rays; Alzheimer's disease, Weight; Cognition; Serum; Mice

\begin{abstract}
Obesity is a severe problem all over the world. One severe problem with obesity is the increased risk of severe diseases. To prevent obesity-related diseases, many people are undertaking prevention measures. Some people try to manage their body weight using supplement drinks, tablets, and health apparatuses. One type of health apparatus is based on the thermal effect. However, the detailed biological mechanisms of the effects of infrared light have not yet been elucidated. In this study, we attempted to clarify the beneficial effects of far infrared light using high-fat diet-treated mice. We exposed mice to far infrared light for 5 months. Body weights were not significantly different in high-fat diet-treated mice that were treated or not treated with far infrared light. However, body weights in far infrared-exposed control mice tended to increase compared to normal mice in the middle treatment term (from 7 to 15 weeks). In the Morris water maze, the goal time of far infrared light-treated control mice was nominally (but not significantly) shorter than the unexposed controls. Serum triglyceride concentrations were significantly increased in far infrared-exposed control mice compared to the unexposed controls. The levels of nerve growth factor and brain-derived neurotrophic factor protein were not significantly different except for nerve growth factor expression in the cerebellum of far infrared-exposed high-fat diet-treated mice. These results indicated that exposure to far infrared light did not provide clear beneficial effects in obese mice. However, the experimental schedule and methods may need to be modified to obtain clearer results.
\end{abstract}

\section{Introduction}

Obesity is a severe problem all over the world, and the percent of obese people is gradually increasing. One severe problem with obesity is an increased risk of secondary severe diseases such as type 2 diabetes mellitus, cardiovascular disease, and high blood pressure [1,2]. In particular, lipid metabolism including liver function is worse in obese people. The values of total cholesterol and triglyceride also significantly increase. On the other hand, calorie restriction extends the lifespan in rodents, C elegans, flies, and humans [3]. One mechanism of lifespan extension due to calorie restriction is oxygen metabolism. Treatment with tocotrienols, which are one type of vitamin E, showed significant anti-obesity effects in our previous studies [4,5]. Several lines of evidence have demonstrated that obesity produces oxidative stress in living tissues and enhances the risks of many diseases [6]. In addition, accumulation of oxidative damage is strongly related to many neurodegenerative disorders, such as Alzheimer's disease (AD) and dementia [7]. To prevent obesity-related diseases, many people undertake prevention measures such as walking, swimming, and food management. Some people try to manage their body weight using supplement drinks, tablets, and health apparatuses. 
One health apparatus is based on the thermal effect and includes infrared (IR) saunas. However, the detailed biological mechanisms of the effect of IR light have not yet been elucidated.

Electromagnetic waves are common in our modern life. Sources include mobile phones, television systems, and medical imaging (X-ray imaging) [8]. Electromagnetic waves, which were discovered by Heinrich Rudolf Hertz in 1887, are classified by different wavelengths and include radio waves, microwaves, IR rays, visible light, ultraviolet, and X-rays. Far IR is a type of IR illumination corresponding to wavelengths ranging from 3 to $1000 \mu \mathrm{m}$. Far IR warms the body, but is not as harmful as ultraviolet, and is widely used in heaters, blankets, bedrock baths, etc. For far IR light, the absorbed IR rays stimulate molecules arranged in bonded lattices, resulting in vibration of molecules, and warming of substances [9]. Far IR light may dilate micro vessels, thereby activating blood circulation and increasing metabolism. We hypothesized that exposure to far IR light enhances blood flow and oxygen metabolism and plays a beneficial role against obesity. The blood flow volume gradually decreases during aging [10] and in AD [11,12]. Decreased blood flow may lead to decreased oxygen levels and malnutrition, accelerating the onset and progression of AD. Here we assessed the effects of far IR light in obese mice using a fabric that emits far IR light.

\section{Materials and Methods}

\section{Animals and Reagents}

All animal experiments were performed with the approval of the Animal Protection and Ethics Committee of the Shibaura Institute of Technology, Tokyo, Japan (Approval number 17006 and 19003). Wild-type C57BL/6 male mice were obtained from
Japan SLC, Inc. (Hamamatsu, Japan) and were maintained until 8or 15 months old. All mice were maintained under conditions of controlled temperature $\left(22 \pm 2^{\circ} \mathrm{C}\right)$, and a 12 -h light/dark cycle, and were provided free access to food and water. Following entry into the study, these mice were maintained for a 5-month period in the presence or absence of a fabric that generates far IR light (Control, $n=5$; Control-Far IR, $n=5$; high-fat diet (HFD), $n=5$; HFD-Far IR, $\mathrm{n}=5$ ). HFD model mice were generated by feeding normal mice a diet (\#D12492; Research Diets Inc., New Brunswick, NJ, USA) containing $5.24 \mathrm{kcal} / \mathrm{g}$, with $60 \%$ of the calories from fat, $20 \%$ from protein, and $20 \%$ from carbohydrates, from 8 months of age until 12 months of age. Control diets (\#D12450J; Research Diets Inc.) containing $3.85 \mathrm{kcal} / \mathrm{g}$, with $10 \%$ of the calories from fat, $20 \%$ from protein, and $70 \%$ from carbohydrates were used for the control groups (Table 1). The body weight and weight of consumed food (food intake) were measured once per week, and the relative body weights (normalized to the starting value for the respective animal) were calculated from the data. After the treatment period, cognition and motor function were assessed using trials, as described below. Following assessments, blood (for serum) was collected from each mouse, mice were euthanized, and samples of brain (cerebral cortex, cerebellum, and hippocampus) were collected for analysis. To assess survival, the mice were maintained until death from natural causes in the presence or absence of a fabric that generates far IR light (Control, $n=10$; Control-Far IR, $n=10$ ). The starting age was 15 months old. Food consisted of normal diet pellets (Labo MR Stock) purchased from Nosan Corp. (Kanagawa, Japan). All other chemical agents were obtained from FUJIFILM Wako Pure Chemical Corp. (Osaka, Japan).

Table 1: Composition of food.

\begin{tabular}{|c|c|c|c|c|}
\hline & \multicolumn{2}{|c|}{ D12492 } & \multicolumn{2}{|c|}{ D12450 } \\
\hline & [g] & [kcal] & [g] & [kcal] \\
\hline Protein & 26 & 20 & 19.2 & 20 \\
\hline Carbohydrates & 26 & 20 & 67.3 & 70 \\
\hline Fat & 35 & 60 & 4.3 & 10 \\
\hline Total & & 100 & & 100 \\
\hline $\mathrm{Kcal} / \mathrm{g}$ & 5.24 & & 3.85 & \\
\hline Casein, 30 mesh & 200 & 800 & 200 & 800 \\
\hline L-Cystine & 3 & 12 & 3 & 12 \\
\hline Corn starch & 0 & 0 & 506.2 & 2024.8 \\
\hline Maltodectrin 10 & 125 & 500 & 125 & 500 \\
\hline Surcose & 68.8 & 275 & 68.8 & 275.2 \\
\hline Cellulose, BW200 & 50 & 0 & 50 & 0 \\
\hline Soybean Oil & 25 & 225 & 25 & 225 \\
\hline Lard & 245 & 2205 & 20 & 180 \\
\hline Mineral Mix & 10 & 0 & 10 & 0 \\
\hline Dicalcium carbonate & 13 & 0 & 13 & 0 \\
\hline Calcium Carbonate & 5.5 & 0 & 5.5 & 0 \\
\hline
\end{tabular}




\begin{tabular}{|c|c|c|c|c|}
\hline Potassium citrate, $1 \mathrm{H} 20$ & 16.5 & 0 & 16.5 & 0 \\
\hline Vitamin Mix V10001 & 10 & 40 & 10 & 40 \\
\hline Choline Bitartrate & 2 & 0 & 2 & 0 \\
\hline FD\&C Blue Dye\#1 & 0.05 & 0 & & \\
\hline FD\&C yellow Dye\#5 & & & 0.04 & 0 \\
\hline Total & 773.85 & 4057 & 1055.05 & 4057 \\
\hline \multicolumn{5}{|c|}{ Mineral Mix S10026 } \\
\hline & [g] & \multicolumn{3}{|c|}{ [Amt in 10g] } \\
\hline \multirow[t]{2}{*}{ Sodium Chloride } & 259 & $1.0 \mathrm{~g}$ & \multicolumn{2}{|r|}{$\mathrm{Na}$} \\
\hline & & $1.6 \mathrm{~g}$ & \multicolumn{2}{|r|}{$\mathrm{Cl}$} \\
\hline Magnesium oxide, Heavy, Dc, USP & 41.9 & $0.5 \mathrm{~g}$ & \multicolumn{2}{|r|}{$\mathrm{Mg}$} \\
\hline Magnesium Sulfate, Heptahydrate & 257.6 & $0.33 \mathrm{~g}$ & \multicolumn{2}{|r|}{$\mathrm{S}$} \\
\hline Ammonium Molybdate Tetrahydrate & 0.3 & $1.6 \mathrm{mg}$ & \multicolumn{2}{|r|}{ Mo } \\
\hline Chromium Potassium Sulfate & 1.925 & $2.0 \mathrm{mg}$ & \multicolumn{2}{|r|}{$\mathrm{Cr}$} \\
\hline Copper carbonate & 1.05 & $6.0 \mathrm{mg}$ & \multicolumn{2}{|r|}{$\mathrm{Cu}$} \\
\hline Ferric Citrate & 21 & $37 \mathrm{mg}$ & \multicolumn{2}{|r|}{$\mathrm{Fe}$} \\
\hline Magnesium carbonate Hydrate & 12.25 & $59 \mathrm{mg}$ & \multicolumn{2}{|r|}{$\mathrm{Mn}$} \\
\hline Potassium lodate & 0.035 & $0.2 \mathrm{mg}$ & \multicolumn{2}{|r|}{ I } \\
\hline Sodium Fluoride & 0.2 & $0.9 \mathrm{mg}$ & \multicolumn{2}{|r|}{ FI } \\
\hline Sodium selenite & 0.035 & $0.16 \mathrm{mg}$ & \multicolumn{2}{|r|}{ Se } \\
\hline Zinc carbonate & 5.6 & $29 \mathrm{mg}$ & \multicolumn{2}{|r|}{$\mathrm{Zn}$} \\
\hline Sucrose & 399.105 & 3.9911 & \\
\hline Total & & & 1000 & \\
\hline \multicolumn{5}{|c|}{ Vitamin Mix V10001 } \\
\hline & & [g] & \multicolumn{2}{|c|}{ [Amt in 10g] } \\
\hline \multicolumn{2}{|c|}{ Vitamin A Acetate(500, 000 IU/g) } & 0.8 & $4000 I U$ & Vitamin A \\
\hline \multicolumn{2}{|c|}{ Vitamin D3(100,000 IU/g) } & 1 & $1000 I U$ & Vitamin D3 \\
\hline \multicolumn{2}{|c|}{ Vitamin E Acetate $(500 \mathrm{IU} / \mathrm{g})$} & 10 & $50 I U$ & Vitamin E \\
\hline \multicolumn{2}{|c|}{ Menadione Sodium Bisulfite(62.5\% Menadoine) } & 0.08 & $0.5 \mathrm{mg}$ & Menadione \\
\hline Biotine $1.0 \%$ & & 2 & $0.2 \mathrm{mg}$ & Bitoine \\
\hline Cyanocobalamin, 0 & & 1 & $10 \mu \mathrm{g}$ & Vitamin B12 \\
\hline Folic Acid & & 0.2 & $2 \mathrm{mg}$ & Folic Acid \\
\hline Nicotine Acid & & 3 & $30 \mathrm{mg}$ & Niacin \\
\hline Calcium Pantothen & & 1.6 & $16 \mathrm{mg}$ & Pantothenic Acid \\
\hline Pyridoxine-HCL & & 0.7 & $7 \mathrm{mg}$ & Vitamin B6 \\
\hline Riboflavin & & 0.6 & $6 \mathrm{mg}$ & Vitamin B2 \\
\hline Thiamin HCL & & 0.6 & $6 \mathrm{mg}$ & Vitamin B1 \\
\hline Sucrose & & 978.42 & & \\
\hline Total & & 1000 & & \\
\hline & ${ }^{*}$ Vita & copherol & & \\
\hline
\end{tabular}

\section{Exposure of Mice to Far IR Light}

Fabric with ores that emit far IR light was a gift from PMC Co., Ltd. (Ishikawa, Japan) (product name: Alpha Slim: U.S. FDA registration number: 30 in09747901). The fabric swatches were replaced once per week throughout the study. To ensure proximity between the fabric and the animals, the volume of crumpled paper, which is typically used as a flooring material, was decreased from the usual amount. Control animal cages were supplied with the same fabric lacking the additive in the ores. In a pilot study, we frequently modified the experimental set-up regarding how to place the fabric in the cages. Each cage also included an enrichment tool.

\section{Cognitive performance}

Cognitive functions were assessed with the Morris water maze and Rota Rod tests, as described previously $[13,14]$ with some 
modifications. Tests were administered using equipment purchased from Muromachi Kikai Co., Ltd. (Tokyo, Japan). The Morris water maze apparatus ( $140 \mathrm{~cm}$ in diameter and $45 \mathrm{~cm}$ in height) consisted of a pool constructed of acrylic resin. The bottom of the pool was divided into four quadrants by lines and was set up with four different visible marks positioned around the pool. A submerged platform was placed in the center of one quadrant. The water temperature of the pool was maintained at $22 \pm 2^{\circ} \mathrm{C}$. Before starting the cognitive performance trials, the animals were acclimated to the pool and to handling by the experimenter by being allowed to swim freely for $60 \mathrm{sec}$ in the absence of a platform and by being handled over a 3-day period. The cognitive trials were performed 4 times per day for 5 consecutive days. All trials were performed at the same time of day and were carried out every 3 hours (starting at 10:00,13:00, 16:00, and 19:00). Thus, we performed a total of 20 trials per mouse. The platform was maintained in the same location in the pool for all trials. The escape latency (time to reach the goal), swimming distance, swimming speed, and proportion of time spent swimming in the quadrant containing the platform were measured using ANY-maze software (version 6.19, Stoelting Co., Wood Dale, IL, USA). For the Rota Rod test, the speed was set to accelerate from 5 to $50 \mathrm{rpm}$ over $120 \mathrm{sec}$, and the time and speed (in rpm) when the animal fell from the Rota Rod were measured. The trials were performed three times and were carried out every $20 \mathrm{~min}$.

\section{Blood Analysis}

Prior to euthanasia, blood was collected without an anticoagulant. Following clotting (approximately $30 \mathrm{~min}$ at room temperature (RT)), the samples were centrifuged in a clinical centrifuge, and the resulting serum supernatants were transferred to fresh tubes, flash frozen, and stored at $-80^{\circ} \mathrm{C}$ until analysis. The samples were assessed for selected clinical chemistry parameters (including total protein, albumin, iron, alanine aminotransferase (ALT), lactic acid dehydrogenase, total cholesterol, and others) using an external inspection agency (Oriental Yeast Co., Ltd., Tokyo, Japan).

\section{Western Blotting}

All brain samples were homogenized in phosphate-buffered saline and used for western blotting as described previously [15], with some modifications. Following homogenization, sample lysates were centrifuged; the resulting supernatants (protein extracts) were used for blotting after determining the protein concentration with the Bradford assay (Bio-Rad protein assay, \#500-0006JA, Bio-Rad Laboratories, Inc., Hercules, CA, USA) according to the manufacturer's protocol. Aliquots of the protein extracts corresponding to $10 \mu \mathrm{g}$ total protein each were separated on $10 \%$ sodium dodecyl sulfate polyacrylamide gels and transferred to nitrocellulose (NC) transfer membranes (ClearTrans; FUJIFILM Wako Pure Chemical Corp.). First, the NC membranes were stained with Ponceau S solution (Merck KGaA, Darmstadt, Germany) and imaged. Next, the NC membranes were washed and incubated in blocking solution (Tris-HCl-buffered saline, $\mathrm{pH}$ 7.6, containing $0.1 \%$ Tween 20 and $2 \%$ non-fat skim milk) for $1 \mathrm{~h}$ at RT. Following blocking, the membranes were washed at RT in Tris-buffered saline containing $0.1 \%$ Tween 20 , and then incubated overnight at $4^{\circ} \mathrm{C}$ with each primary antibody. The primary antibodies were as follows: rabbit recombinant monoclonal anti-brain-derived neurotrophic factor (BDNF) antibody [EPR1292], 1:2500 (\#ab108139, Abcam plc., Cambridge, UK); rabbit polyclonal anti-nerve growth factor (NGF) antibody (H-20), 1:500 (\#sc-548, Santa Cruz Biotechnology Inc. (SCBT), Dallas, TX, USA); rabbit polyclonal anti-tropomyosin receptor kinase (Trk)A antibody (763), 1:3200 (\#sc-118, SCBT); and rabbit polyclonal anti-TrkB antibody (H-181), 1:250 (\#sc-8316, SCBT).

The NC membranes then were incubated for $1 \mathrm{~h}$ at RT with the secondary antibody (horseradish peroxidase-conjugated antirabbit immunoglobulin G antibody (Promega Corp., Madison, WI, USA) at 1:4000). All western blotting experiments were performed at least three times. All chemiluminescent signals were generated by incubation with the detection reagents (Immobilon; Merck KGaA) according to the manufacturer's protocol. The relative intensities were determined using an LAS-3000 imaging system (FUJFILM Wako Pure Chemical Corp.). Expression ratios were calculated against those of the Ponceau S staining intensities using Image J software 1.53a. (National Institutes of Health, Bethesda, MD, USA)

\section{Statistical Analysis}

Data are expressed as means \pm standard deviation and were analyzed by using JMP 15.0 software (SAS Institute Japan Inc., Tokyo, Japan). $\mathrm{p}$ values of less than 0.05 were considered statistically significant. Each detailed statistical method is described in the Figure captions.

\section{Results}

\section{Far IR Irradiation Does not Influence Body weight Gain in HFD-Treated Mice}

To clarify the biological effects of far IR light, we measured the body weight of HFD-treated mice housed in the presence or absence of the fabric that generates far IR light. The relative body weight gain (normalized to baseline) of HFD-treated mice was not attenuated in animals housed with the fabric that generates far IR light (compared to those housed with control fabric) (Figure 1). However, the ratio of body weight gain in far IR-exposed control mice nominally (but not significantly) increased in the middle treatment term (from 7 to 15 weeks) compared to the unexposed controls. Food intake weight of both HFD-treated groups significantly differed compared to both control groups (Figure 2). However, calorie intake did not differ among any animal groups. 


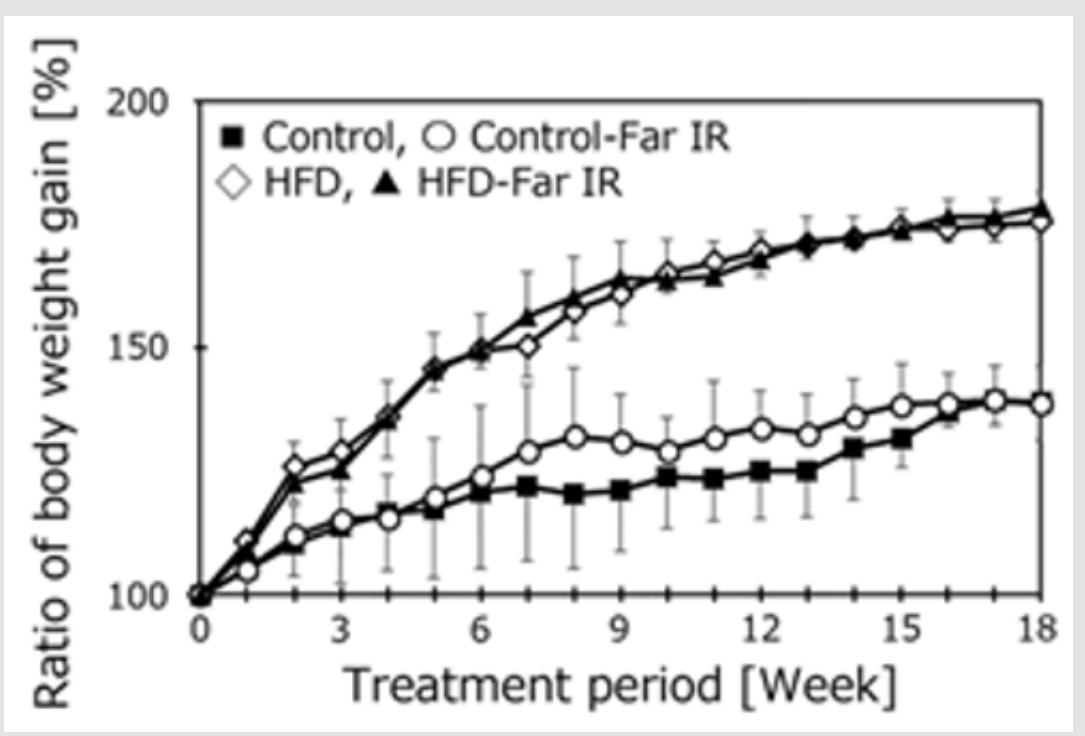

Figure 1: Relative body weights in treatment groups. Body weight was measured once per week for 5 consecutive months. Values were normalized to baseline (defined as 100\%). Data are expressed as the mean \pm SD (Control, $n=5 ;$ Control-Far IR, $n=$ 5; HFD, $n=5$; HFD-Far IR, $n=5$ ). The timeline shows the treatment period. Statistical analysis was performed using two-way analysis of variance (ANOVA). No significant differences were found between the presence or absence of Far IR in the four groups.

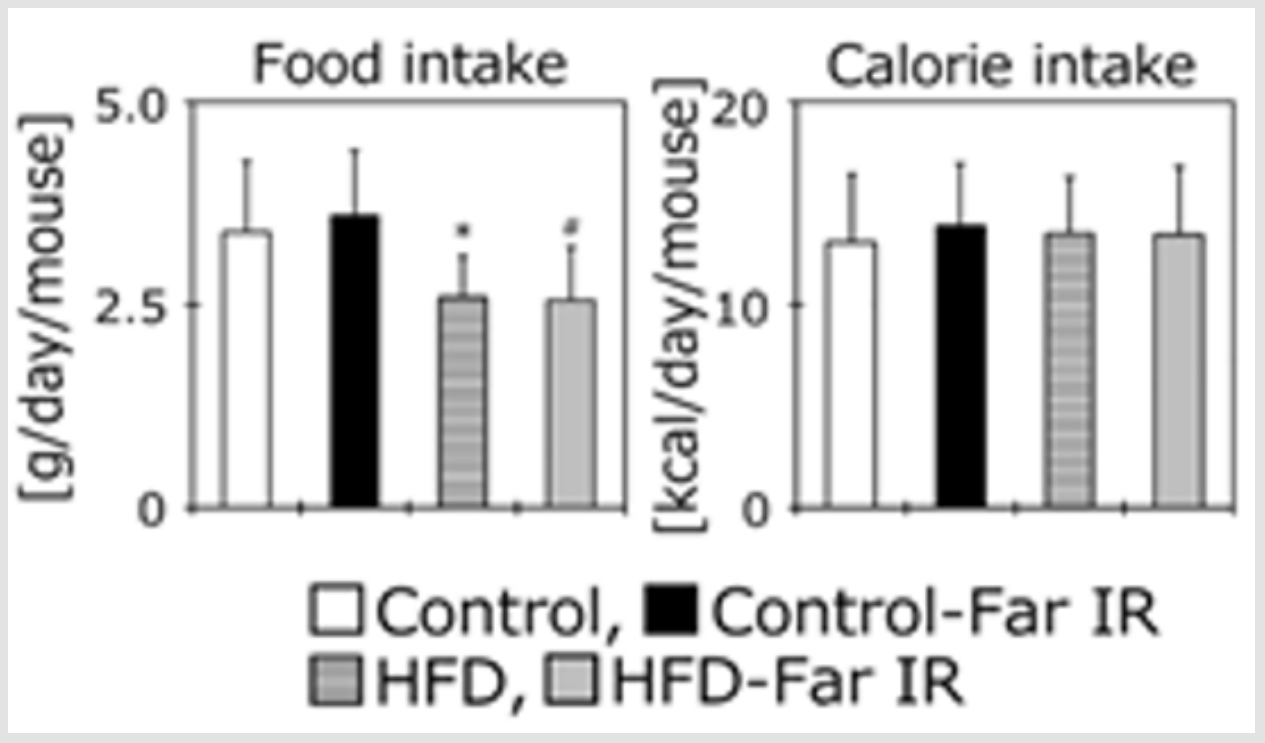

Figure 2: The daily average food and calorie intake of each mouse group. Food intake was measured once per week. These data show the daily average volume (g) for the entire 5-month period. Daily intake of calories was calculated according to food intake. Data re expressed as the mean $+\mathrm{SD}$ (Control, $n=5$; Control-Far IR, $n=5 ; \mathrm{HFD}, \mathrm{n}=5$; HFD-Far IR, $n=5$ ). The comparison was performed using the Tukey-Kramer test. $\left({ }^{*}, \#, p<0.01\right)$.

\section{Measurement of Cognitive Function of HFD-Treated Mice}

\section{in the Presence or Absence of Far IR Light}

To clarify the effect of far IR light on the cognitive function of HFD-treated mice, 5 months after starting housing in a specific cage, we measured the learning ability using the Morris water maze task (Figure 3). The mice were subjected to swim tests 4 times per day on 5 consecutive days. The time to achieve the goal did not differ significantly for the HFD-treated mice housed in the presence or absence of far IR light. However, the goal time in far IR lightexposed control mice tended to be faster in the third and fourth trial days compared to the other three groups. The swimming distance, swimming speed, and percent of time staying in the platform area were not different among the groups. Motor function was assessed with the Rota Rod test (Figure 4). No difference was found in the presence or absence of far IR light. 
(A)

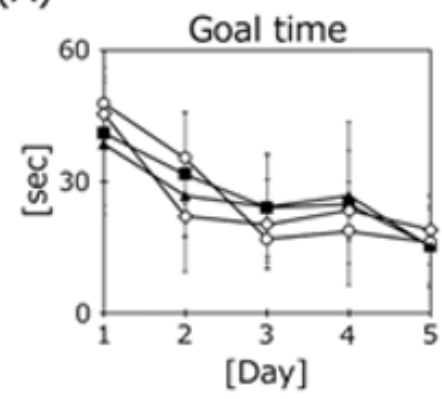

(C)

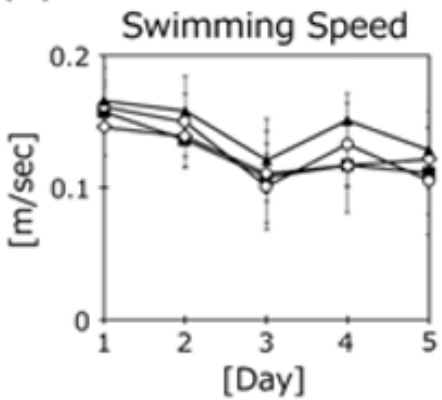

(B)

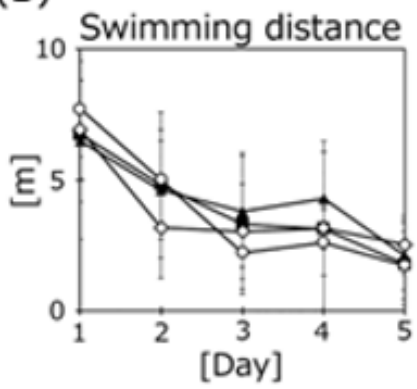

(D)

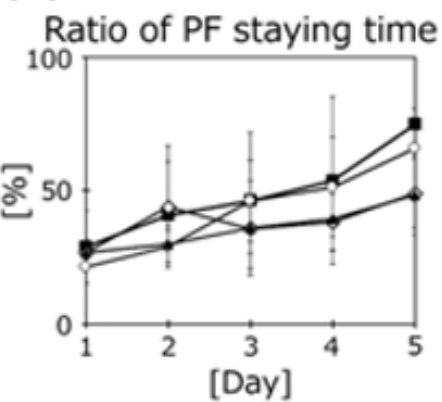

- Control, O Control-Far IR

$\checkmark$ HFD, $\boldsymbol{\Delta}$ HFD-Far IR

Figure 3: Assessment of spatial learning and memory in treatment groups using the Morris water maze test. Data are expressed as the mean + SD (Control, $n=5$; Control-Far IR, $\mathrm{n}=5$; HFD, $n=5$; HFD-Far IR, $n=5)$. All data were compared using two-way ANOVA.

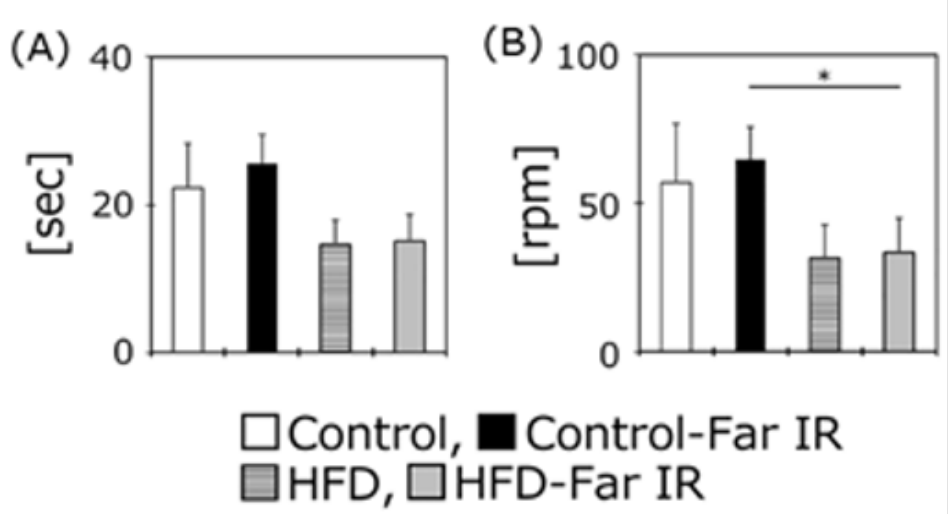

Figure 4: Assessment of motor function in treatment groups using the Rota Rod test. Data are expressed as the mean $+\mathrm{SD}$ (Control, $n=5$; Control-Far IR, $\mathrm{n}=5$; HFD, $n=5$; HFD-Far IR, $n=5$ ). All data were compared using the Tukey-Kramer test. ${ }^{*} \mathrm{p}$ $<0.05$.

\section{Changes in Serum Parameters of HFD-Treated Mice in}

\section{the Presence or Absence of Far IR Exposure}

Exposure to far IR light affects blood flow [16]. After the cognitive trials, the mice were bled, euthanized, and various regions of the brain were recovered. The resulting sera were tested for multiple clinical chemistry parameters (Table 2). Some parameters significantly changed in HFD-treated mice compared to the controls without far IR light. The level of triglyceride in far IR light-exposed controls was significantly increased compared to the unexposed controls. The level of ALT was significantly increase in far IR lightexposed HFD-treated mice compared to the unexposed HFD mice. The level of triglyceride in far IR light-exposed HFD-treated mice showed a nominal (but not significant) decrease compared to the unexposed HFD mice. 
Table 2: Changes in serum parameters for HFD mice maintained in the presence or absence of far IR light.

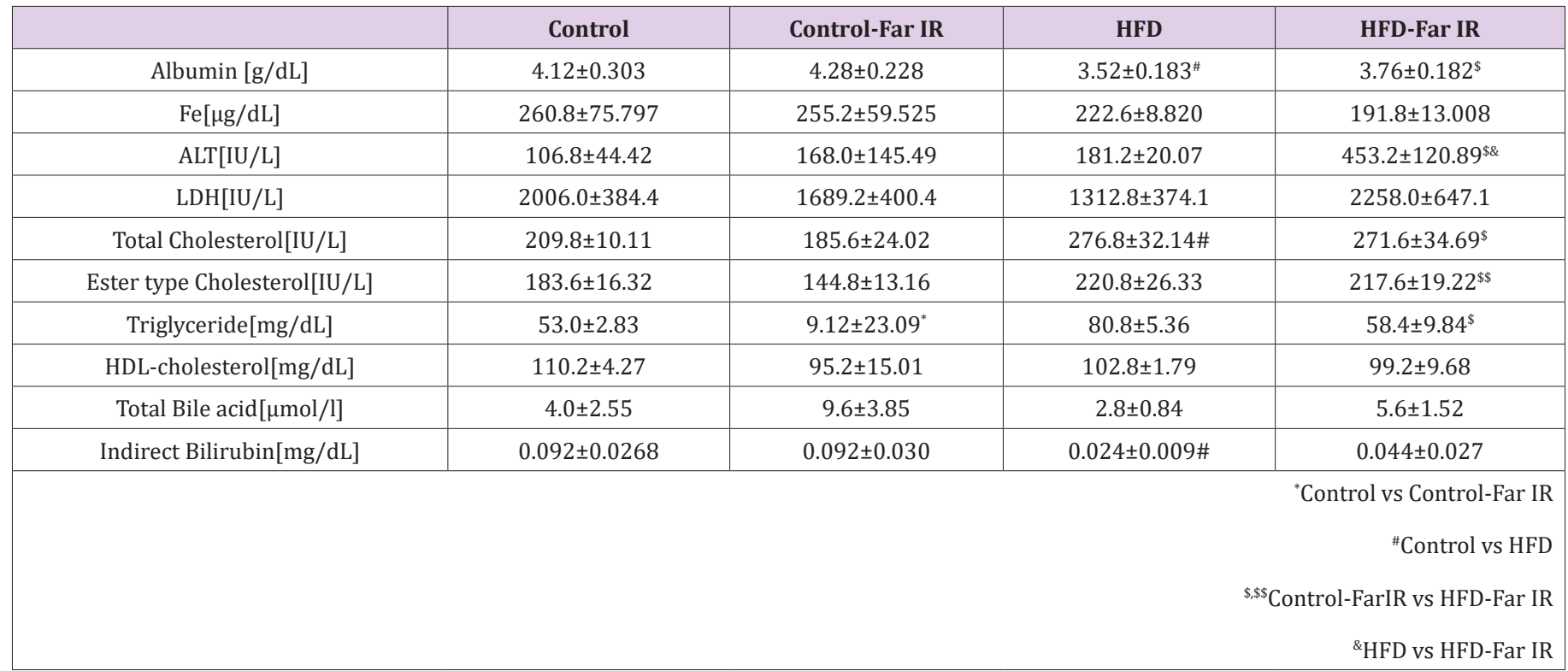

Far IR Exposure did not Influence Neurotrophic Factor Protein Levels in the Brains of HFD-Treated Mice

Neurotrophic factors such as NGF and BDNF play an important role in the maintenance of brain functions. Although the results of cognitive and motor functions were not different among groups, the levels of these factors and of their respective receptors (TrkA and TrkB) were assessed in the cerebral cortex, cerebellum, and hippocampus using western blotting (Figure 5). The NGF protein level in the cerebellum was significantly higher in the far IR-exposed HFD mice than in the HFD controls. However, the other proteins did not differ significantly in any brain regions in the presence or absence of far IR light.

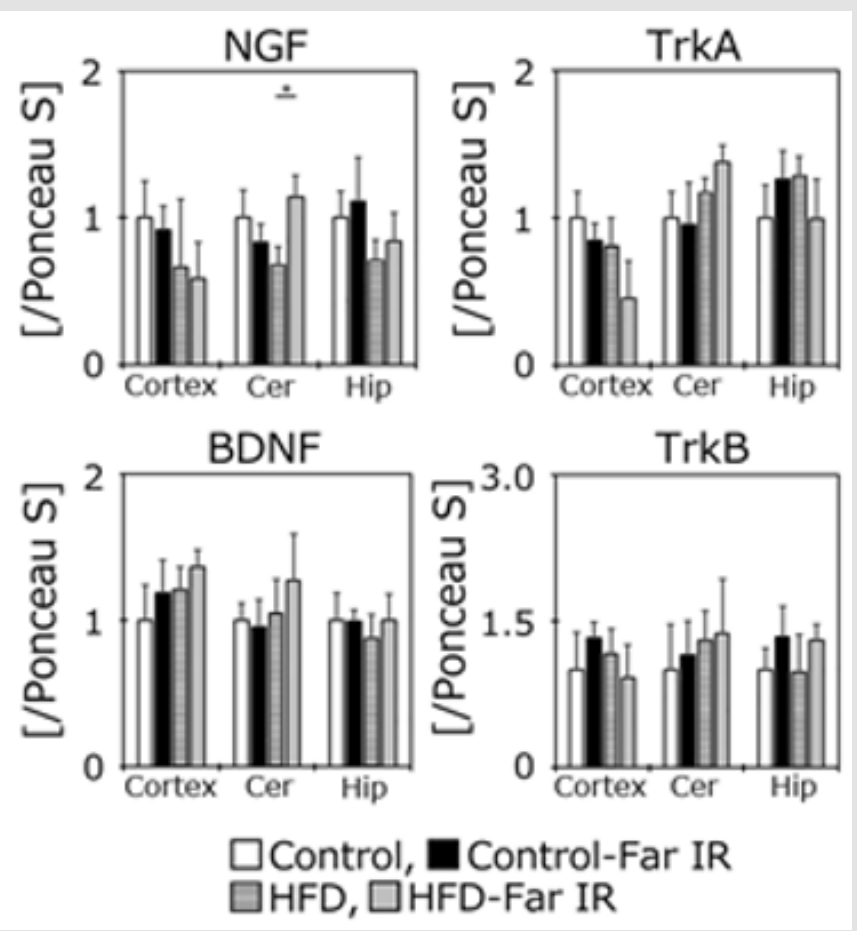

Figure 5: Western blotting analysis of the levels of each protein. Histograms indicate the levels of each protein examined in the cortex (Cortex), cerebellum (Cer), and hippocampus (Hip) obtained from western blots. Data are expressed as the mean \pm SD (Control $(n=5)$, Control-Far IR $(n=5)$. HFD $(n=5)$, HFD-Far IR $(n=5))$. The data were analyzed using the Tukey-Kramer test. ${ }^{*} p<0.05$. 
Far IR Light did not Extend the Life Span in the Control Mice

Finally, we measured longevity in the presence or absence of far IR light on control mice (Figure 6). Longevity was not different according to treatment or no treatment with far IR light in the control mice.

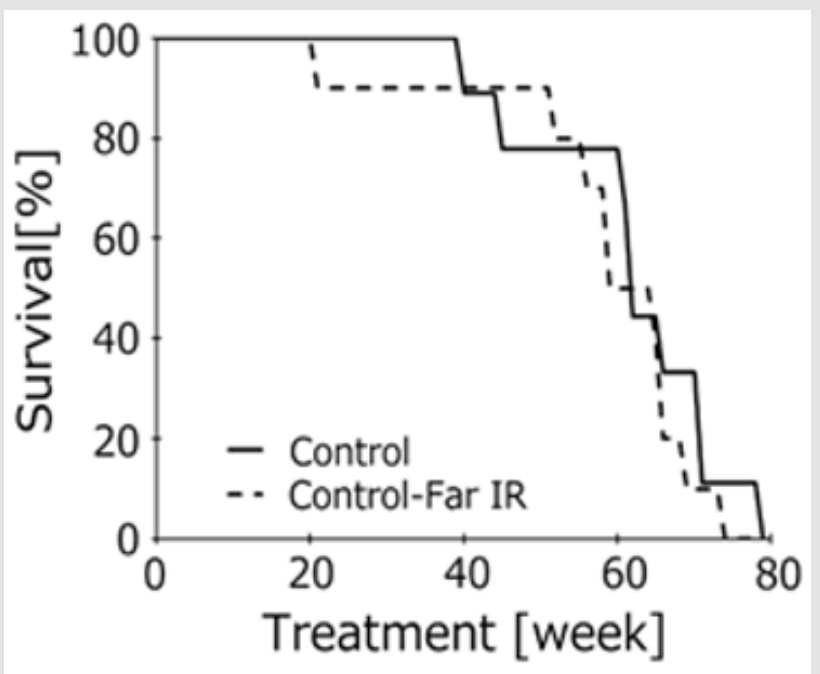

Figure 6: Survival rate of mice housed in the presence or absence of the fabric that generates far IR light. Data are expressed as the mean \pm SD (Control $(n=10)$, Control-Far $\operatorname{IR}(\mathrm{n}=10))$.

\section{Discussion}

\section{Far IR Light Exposure Inhibits Body Weight Gain in Mice}

Far IR light is used in many places in everyday life, such as leg hyperthermia, saunas, cooking, and heat therapy [17]. These are all indispensable in our daily life, but the mechanisms of far IR light effects remain unclear. In the present study, to clarify the potential health benefits of far IR light, we exposed C57BL/6 mice to far IR light for a long-term period (5 months). Specifically, we assessed the effects of far IR light on HFD mice, given that far IR exposure may affect blood flow and change the oxygen concentration in small vessels in the brain. Lipid metabolism may be changed by obesity, and high levels of triglyceride and cholesterol may increase the risk of arteriosclerosis in small vessels. In fact, treatment of mice with the same HFD for 2 months significantly increased the total serum cholesterol level in our previous study [4]. However, contrary to expectations, the percent body weight gain in HFD mice did not differ after far IR light exposure in this experiment. On the other hand, the percent body weight gain in the control mice showed a nominal but not significant difference in the presence compared to the absence of far IR light (from 7 to 15 weeks). The percent body weight gain in the final week was almost the same between the two control groups. The reason for no difference is unknown. We did not observe a clear anti-obesity effect in HFD-treated mice using far IR light in this study. The reason may depend on the diet composition.

The HFD provides excess nutrition to mice. Far IR light, which is non-contact, may not have a sufficient anti-obesity effect in our experimental model. Previously, we examined the anti-obesity effect of tocotrienols, which are natural lipophilic vitamins, using a similar time schedule and the same HFD [5]. Treatment with tocotrienols significantly inhibited body weight gain in the first half of the treatment period. However, the anti-obesity effect of tocotrienols disappeared in the second half of the treatment period. In fact, in the control diet compared to the HFD, the nutritional components are different from a normal pellet diet. The percent body weight gain using a normal pellet diet did not differ from 8 to 12 months (data not shown). This result indicates that treatment with a longterm control diet may also provide excess nutrition to mice. We may have compared four mouse groups with an excess nutrition status. Another reason for this problem may be the breeding environment of the mice. Washington et al. reported that ceramic particles absorb heat emitted from the body, and then re-emit the associated thermal energy back into the body as IR radiation [18]. Thus, we thought that the ceramic would absorb the heat released from the mouse itself and release it to the mouse again to obtain a beneficial effect. However, anti-obesity results were not seen. Although mice prefer cooler conditions of approximately $23^{\circ} \mathrm{C}$, warming the cage floor to approximately $37^{\circ} \mathrm{C}$ may be important. To clarify the antiobesity effect of far IR light, we will perform additional studies using modified methodology.

\section{Far IR Exposure Does not Affect Cognitive Function and Brain Neurotrophic Factors}

To clarify the effects of far IR light on spatial memory in HFDtreated mice, we measured learning ability using the Morris water maze task. This test measures the time taken by mice to reach a hidden acrylic platform $[13,14]$. The goal times did not differ significantly between the control and HFD-treated mice housed in the presence or absence of far IR light, although the goal times on the third and fourth days of testing were nominally (but not significantly) lower in the far IR-exposed control mice compared to the unexposed controls. The swimming speed, distance, and percent time spent near the platform did not differ among all groups, nor did motor function as assessed using the Rota Rod apparatus. These results showed that far IR exposure did not influence cognitive function in HFD-treated mice. The scores on the Rota Rod were strongly related to body weight. Additionally, some mice were floating, but not swimming in the pool during trials. Neither test may be suitable for the HFD mouse model. We want to try different maze apparatuses such as the open field, Y-maze, and Barns maze. Because clear results were not obtained in either test, we checked the protein expression of brain neurotrophic factors because we hypothesized that oxidation was promoted in the HFD- 
treated mouse brains. Accumulation of oxidative damage induces cognitive dysfunction and increases the risk of neurodegenerative disorders [19].

BDNF and NGF play important roles in the maintenance of brain function including cognition [20,21]. However, the protein expression of NGF, BDNF, and their receptors were not different among groups, except for NGF expression in the cerebellum of far IR-exposed HFD-treated mice. The cause of the disappointing result may be related to the difference in brain oxidation levels. We fed the mice a HFD for a relatively long period (5 months). However, our HFD does not contain high carbohydrates. Some researchers use a high-fat and high carbohydrate diet and then measure antioxidant effects such as vitamin E [22]. Although our diet provided excess nutrition, it may be insufficient for accelerating oxidation in the mouse's body. Another possible reason for this problem may be the animal species. Fernando et al. [23] reported that treatment of Sprague Dawley rats with a HFD for 2 months induces cognitive dysfunction via attenuation of hippocampal BDNF mRNA and protein expression, and that this dysfunction is improved by vitamin $\mathrm{E}$ treatment. Exposure of Wistar rats to a high oxygen concentration for 48 hours significantly increases brain lipid peroxidation levels and induces cognitive dysfunction as seen in the Morris water and radial arm mazes [13]. We suggest that mice have relatively strong resistance against oxidative stress compared to rats. Thus, modification of food components and the animal species may be needed for detection of a clear result following exposure to far IR light.

We found no significant difference in survival of control mice housed in the presence or absence of far IR light. The purpose of this study was to determine if far IR light suppresses body weight gain, affects lipid metabolism, and reduces oxidative stress production, all of which may extend the lifespan. In calorie restriction research, the lifespan is extended by changes in lipid metabolism and oxygen consumption [24]. We will assess animal survival using the obesity model soon.

\section{Conclusion}

In this study, we examined the possible biological effects of long-term exposure of HFD mice to far IR light. Notably, neither cognition nor motor function in HFD mice was improved after far IR exposure in our experimental models. However, body weight, some serum parameters, and brain neurotrophic factor protein expression changed by a small amount. Far IR light clearly plays a beneficial role in our everyday life including in the medical field. In the near future, we want to provide evidence to clarify the beneficial mechanism of far IR light.

\section{Author Contributions}

Conceptualization, K.F. and M.K.; Data analysis \& resources, Y.K., M.K., and K.F.; all other contributions to the research, K.F.

\section{Acknowledgments}

This work was supported by the Ministry of Education, Culture, Sports, Science, and Technology (MEXT)-Supported Program for the Strategic Research Foundation at Private Universities.

\section{References}

1. Expert Panel Members, Jensen MD, Ryan DH, Donato KA, Apovian CM, Ard JD, et al. (2013) Executive summary: Guidelines (2013) for the management of overweight and obesity in adults: A report of the American College of Cardiology/American Heart Association Task Force on Practice Guidelines and the Obesity Society published by the Obesity Society and American College of Cardiology/American Heart Association Task Force on Practice Guidelines. Based on a systematic review from the Obesity Expert Panel. Obesity (Silver Spring) 22 (Suppl. 2): S5-S39.

2. The GBD 2015 Obesity Collaborators Health effects of overweight and obesity in 195 countries over 25 years. N Eng J Med 377: 13-27.

3. Ingram DK, de Cabo R (2017) Calorie restriction in rodents: Caveats to consider. Ageing Res Rev 39: 15-28.

4. Fukui K, Shirai M, Ninuma T, Kato Y (2019) Anti-obesity effects of tocotrienols and bran in high-fat diet-treated mice. Nutrients 11(4): 830.

5. Kato Y, Aoki Y, Fukui K (2020) Tocotrienols influence body weight gain and brain protein expression in long-term high-fat diet-treated mice. Int J Mol Sci 21(12): 4533.

6. Jimenez Gomez Y, Mattison JA, Pearson KJ, Martin Montalvo A, Palacious HH, et al. (2013) Resveratrol improves adipose insulin signaling and reduces the inflammatory response in adipose tissue of rhesus monkeys on high-fat, high-sugar diet. Cell Metab 18: 533-545.

7. Butterfield DA (2014) The 2013 SFRBM discovery award: Selected discoveries from the butterfield laboratory of oxidative stress and its sequela in brain in cognitive disorders exemplified by Alzheimer disease and chemotherapy induced cognitive impairment. Free Radic Biol Med 74: 157-174.

8. Vatansever F, Hamblin MR (2012) Far infrared radiation (FIR): its biological effects and medical applications. Photonics Lasers Med 4: 255-266.

9. Lee MS, Baletto F, Kanhere DG, Scandolo S (2008) Far-infrared absorption of water clusters by first-principles molecular dynamics. J Chem Phys 128(21): 214506.

10. Tarumi T, Zhang R (2018) Cerebral blood flow in normal aging adults: cardiovascular determinants, clinical implications, and aerobic fitness. J Neurochem 144(5): 595-608.

11. Zhang N, Gordon ML, Goldberg TE (2017) Cerebral blood flow measured by arterial spin labeling MRI at resting state in normal aging and Alzheimer's disease. Neurosci Biobehav Rev 72: 168-175.

12. Willette AA, Kapogiannis D (2015) Does the brain shrink as the waist expands? Ageing Res Rev 20: 86-97.

13. Fukui K, Onodera K, Shinkai T, Suzuki S, Urano S (2001) Impairment of learning and memory in rats caused by oxidative stress and aging, and changes in antioxidative defense systems, Ann N Y Acad Sci 928: 168175 .

14. Fukui K, Omoi N, Hayasaka T, Shinnkai T, Suzuki S, et al. (2002) Cognitive impairment of rats caused by oxidative stress and aging, and its prevention by vitamin E. Ann N Y Acad Sci 959: 275-284.

15. Fukui K, Nakamura K, Shirai M, Takatsu H, Urano S, et al. (2014) Longterm vitamin E-deficient mice exhibit cognitive dysfunction via elevation of brain oxidation, Free Radic Res 48(6): 649-658.

16. Wan Q, Yang S Li L, Chu F (2017) Effects of far infrared therapy on arteriovenous fistulas in hemodialysis patients: a meta-analysis, Ren Fail 39(1): 613-622. 
17. Fukui K, Ostapenko VV, Abe K, Nishide T, Miyano M, et al. (2006) Changes in plasma alpha and gamma tocopherol levels before and after long-term local hyperthermia in cancer patients, Free Radic Res 40(8): 893-899.

18. Washington K, Wason J, Thein MS, Lavery LA, Hamblin MR, et al (2018) Randomized controlled trial comparing the effects of farinfrared emitting ceramic fabric shrts and control polymer shirts on transcutaneous P02. J Text Sci Eng 8(2): 349.

19. Fukui K (2016) Reactive oxygen species induce neurite degeneration before induction of cell death. J Clin Biochem Nutr 59(3): 155-159

20. Nagahara AH, Merrill DA, Coppola G, Tsukada S, Schroeder BE, et al. (2009) Neuroprotective effects of brain-derived neurotrophic factor in rodent and primate mdels of Alzheimer's disease. Nat Med 15(3): 331337.

ISSN: 2574-1241

DOI: 10.26717/BJSTR.2021.33.005468

Koji Fukui. Biomed J Sci \& Tech Res

(C) This work is licensed under Creative

Submission Link: https://biomedres.us/submit-manuscript.php
21. Chen AI, Zang K, Masliah E, Reichardt LF (2016) Glutamatergic axonderived BDNF controls GABAergic synaptic differentiation in the cerebellum. Sci Rep 6: 20201.

22. Karem HA, Omar FK, Heba AS, Zuheir H (2013) Vitamin E prevents highfat high-carbohydrates diet-induced memory impairment: The role of oxidative stress. Physiol Behav 119: 72-78.

23. Wu A, Ying Z, Gomez Pinilla F (2004) The interplay between oxidative stress and brain-derived neurotrophic factor modulates the outcome of a saturated fat diet on synaptic plasticity and cognition. Eur J Neurosci 19: 1699-1707.

24. Mattison JA, Roth GS, Beasley TM, Tilmont EM, Handy AM, et al. (2012) Impact of calorie restriction on health and survival in rhesus monkeys from the NIA study. Nature 489: 318-321.

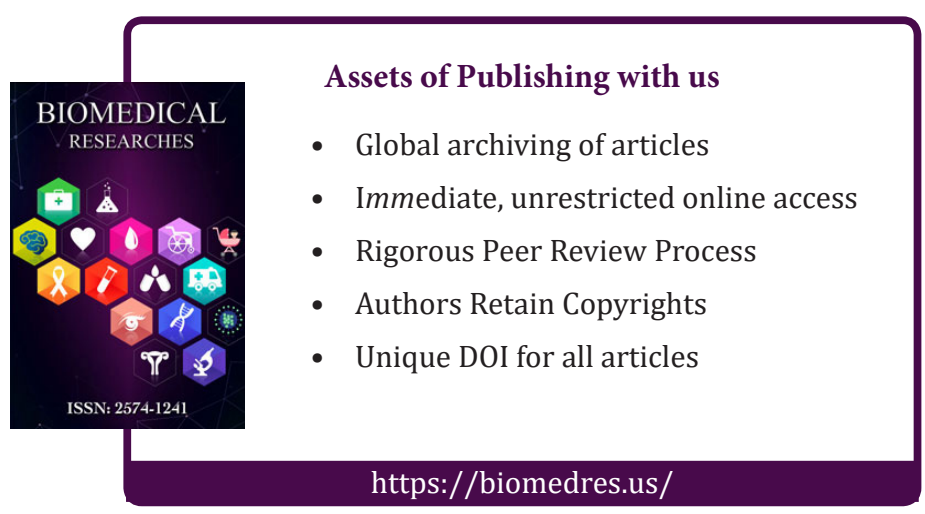

